Modern Physics Letters A,

(C) World Scientific Publishing Company

\title{
A Model of Two Dimensional Turbulence Using Random Matrix Theory
}

\author{
Savitri V. Iyer \\ State University of New York at Geneseo \\ Geneseo, NY 14454, USA \\ S.G. Rajeev \\ University of Rochester \\ Rochester, NY 14627, USA \\ Received (received date) \\ Revised (revised date)
}

\begin{abstract}
We derive a formula for the entropy of two dimensional incompressible inviscid flow, by determining the volume of the space of vorticity distributions with fixed values for the moments $Q_{k}=\int \omega(x)^{k} d^{2} x$. This space is approximated by a sequence of spaces of finite volume, by using a regularization of the system that is geometrically natural and connected with the theory of random matrices. In taking the limit we get a simple formula for the entropy of a vortex field. We predict vorticity distributions of maximum entropy with given mean vorticity and enstrophy; also we predict the cylindrically symmetric vortex field with maximum entropy. This could be an approximate description of a hurricane.
\end{abstract}

\section{Introduction}

Two dimensional inviscid incompressible flow is completely determined by solutions to the Euler equations in the absence of random external forces. Detailed numerical as well as analogue simulations 1 show complex phenomena such as recombination of vortices. Still, the situation is much simpler than in three dimensions. It appears that energy flows from small scales to large scales so that over long time intervals the flow becomes smoother. This is the opposite of the behavior observed in three dimensional hydrodynamics. Thus two dimensional flow is not turbulent in the same sense as three dimensional flow. Nevertheless, there is sufficient complexity remaining even in two dimensions that a statistical approach is worthwile.

The study of the statistical mechanics of inviscid flow dates back to Onsager with some recent revivals 3 . The crucial input into any statistical theory is the formula for entropy. Previous studies have used various postulates for the entropy of a vortex field $\omega(x)$ such as

$$
\int \omega(x) \log |\omega(x)| d^{2} x
$$


We will determine the formula for entropy predicted by its microscopic (Boltzmann) definition as the logarithm of the volume of the phase space with a fixed value of the conserved quantities (more precisely, central observables - see below). The conserved quantities of the Euler equation are the moments $Q_{k}=\int \omega(x)^{k} d^{2} x$. The volume of the phase space is difficult to determine directly because it is infinite dimensional.

We will determine a formula for entropy by 'regularizing' the system; i.e., approximating it with a finite dimensional system. The volume of the phase space is then finite -indeed the answer is known in the literature on Random Matrix theory . The entropy of the original system can then be determined by taking the limit as the dimension goes to infinity. We establish this way that the formula for entropy is

$$
\chi=\frac{1}{A^{2}} \int \log |\omega(x)-\omega(y)| d^{2} x d^{2} y
$$

where $A$ is the area of the region within which the flow is contained. This is quite different from the postulates used in earlier analyses formula to predict the maximum entropy configuration with given mean vorticity and enstrophy* - it is the Wigner semi-circular distribution. We predict the vorticity distribution of an axi-symmetric vortex of maximum entropy.

Polyakor 6 has presented another theory of two dimensional turbulence, based on the group of conformal transformations. Our considerations are in many ways orthogonal, being based on the group of area preserving transformations rather than conformal transformations (which preserve instead the angles).

In Section 2 we present an overview of two dimensional hydrodynamics. The Euler equations are formulated as a hamiltonian dynamical system, in analogy to the rigid body equations also due to Euler.

In Section 3 we discuss the regularization procedure. We use ideas that have their origin in quantum field theory, but have already been used in the hydrodynamics literatured.

In Section 4 we reformulate the regularized system in terms of hermitean matrices which allows us to use the ideas from random matrix theory to derive a formula for entropy in Section 5. In Section 6 we derive the distribution function for vorticity that maximizes entropy for given mean vorticity and enstrophy. And finally we predict the axi-symetric vortex field of maximum entropy in Section 7.

\section{Two-Dimensional Hydrodynamics as a Hamiltonian System}

Incompressible hydrodynamics is described by the Navier-Stokes equation:

$$
\frac{D \mathbf{u}}{D t}=-\nabla p+R^{-1} \Delta \mathbf{u}+\mathbf{f}
$$

along with the constraint $\nabla \cdot \mathbf{u}=0$. Here, $\mathbf{u}, p, R, \mathbf{f}$ are the velocity field, the pressure, the Reynolds number, and the external force field respectively. We are

*Enstrophy is the second moment of vorticity: $Q_{2}=\int \omega^{2}(x) d^{2} x$. 
using units in which the mass density of the fluid is equal to one. Also, the total derivative is defined by

$$
\frac{D a}{D t}=\frac{\partial a}{\partial t}+\mathbf{u} \cdot \nabla a
$$

Pressure can be eliminated by taking the curl of this equation. This gives

$$
\frac{D \boldsymbol{\omega}}{D t}=\boldsymbol{\omega} \cdot \nabla \mathbf{u}+R^{-1} \Delta \boldsymbol{\omega}+\nabla \times \mathbf{f}
$$

where $\boldsymbol{\omega}=\nabla \times \mathbf{u}$ is the vorticity.

Note that for incompressible flow, vorticity determines velocity:

$$
u_{a}(x)=\epsilon_{a b c} \partial_{b} \int \omega_{c}(y) G(x, y) d^{3} y
$$

or

$$
u_{a}(x)=\epsilon_{a b} \partial_{b} \int \omega(y) G(x, y) d^{2} y,
$$

in two dimensions. Here $G(x, y)$ is the Green's function of the Laplace operator $\Delta_{x} G(x, y)=-\delta(x-y)$ with the appropriate boundary conditions on vorticity. For example, if $\boldsymbol{\omega} \rightarrow 0$ as $|x| \rightarrow \infty, G(x, y)=-\frac{1}{4 \pi|\boldsymbol{x}-\boldsymbol{y}|}$ in three dimensions and $G(x, y)=-\frac{1}{2 \pi} \log |x-y|$ in two dimensions.

If the flow is two dimensional (i.e., $\boldsymbol{u}$ is independent of the $z$ coordinate), vorticity is orthogonal to the $x y$-plane so that $\boldsymbol{\omega} \cdot \nabla \mathbf{u}=0$. Moreover vorticity can be thought of as a pseudo-scalar. Thus, for two dimensional incompressible inviscid (i.e., $R^{-1} \rightarrow 0$ ) flow without external forces, vorticity is conserved along flow lines, with

$$
\frac{D \omega}{D t}=0, \quad \omega=\epsilon_{a b} \partial_{a} u_{b} .
$$

By expressing velocity in terms of vorticity we get an integro-differential equation:

$$
\frac{\partial \omega}{\partial t}=\partial_{a} \omega \epsilon_{a b} \partial_{b} \int G(x, y) \omega(y) d^{2} y .
$$

These are analogous to another system studied by Euler: the equations for the rigid body given by

$$
\frac{d L_{a}}{d t}=\epsilon_{a b c} L_{b} A_{c d} L_{d}
$$

where $A_{a b}$ is the inverse of the moment of inertia. The integral operator on the rhs is analogous to the inverse of moment of inertia. Thus vorticity corresponds to angular momentum, the stream function to angular velocity and the Laplace operator to the moment of inertia. The deep reason for this analogy describe equations for geodesics on a group manifold. In the case of the rigid body,

${ }^{a}$ For three dimensional flow, vorticity is a vector field. Inviscid flow without external forces satisfies $\frac{\partial \boldsymbol{\omega}}{\partial t}+\mathcal{L} \boldsymbol{u} \boldsymbol{\omega}=0$, where the Lie derivative of vector fields is $\mathcal{L}$ is given by $\mathcal{L} \boldsymbol{u} \boldsymbol{\omega}=\boldsymbol{u} \cdot \nabla \boldsymbol{\omega}-\boldsymbol{\omega} \cdot \nabla \boldsymbol{u}$. Thus, vorticiy is still conserved by the flow. 
it is the rotation group with the metric given by the moment of inertia tensor; in the case of hydrodynamics, it is the group of area preserving diffeomorphisms with the $L^{2}$-metric. In more physical terms, this analogy can be seen in the hamiltonian formalism.

Recall that the space of functions on the plane is a Lie algebra, with the Lie bracket given by:

$$
\left[f_{1}, f_{2}\right]=\epsilon^{a b} \partial_{a} f_{1} \partial_{b} f_{2} .
$$

Every function $f: R^{2} \rightarrow R$ corresponds to an observable of two dimensional hydrodynamics, $\omega_{f}=\int f(x) \omega(x) d^{2} x$. The above Lie bracket of functions suggests a natural postulate of Poisson bracket for vorticity:

$$
\{\omega(x), \omega(y)\}=\epsilon^{a b} \partial_{b} \omega(x) \partial_{a} \delta(x-y) ;
$$

so that, $\left\{\omega_{f_{1}}, \omega_{f_{2}}\right\}=\omega_{\left[f_{1}, f_{2}\right]}$. The natural postulate for the hamiltonian is the total energy of the fluid,

$$
H=\frac{1}{2} \int \boldsymbol{u}^{2} d^{2} x=\frac{1}{2} \int G(x, y) \omega(x) \omega(y) d^{2} x d^{2} y .
$$

A straightforward calculation shows that this hamiltonian with the above Poisson bracket for vorticity indeed gives the equation of motion of two dimensional hydrodynamics.

This is analogous to the hamiltonian formalism of rigid body mechanics. The Poisson bracket of the angular momentum (as measured in the co-moving coordinates) arises from the Lie algebra of infinitesimal rotations,

$$
\left\{L_{a}, L_{b}\right\}=\epsilon_{a b c} L_{c} .
$$

The hamiltonian is the rotational kinetic energy

$$
H=\frac{1}{2} A_{a b} L_{a} L_{b},
$$

where $A$ is the inverse of the moment of inertia matrix. Thus, vorticity is analogous to angular momentum; the Lie algebra of functions on the plane analogous to the Lie algebra of rotations; and the Green's function of the Laplace operator analogous to the inverse of the moment of inertia matrix.

The square of the angular momentum $L^{2}=L_{a} L_{a}$ is a central function (i.e., has zero Poisson bracket with all functions of angular momentum). In particular it commutes with the hamiltonian and hence is conserved. The phase space of rigid body mechanics is the sphere on which $L^{2}$ is constant: this is the 'symplectic leaf' of the Poisson algebra.

Analogously, the moments

$$
Q_{k}=\int \omega^{k}(x) d^{2} x, \quad k=1,2, \cdots
$$


are central functions (hence conserved quantities) in two dimensional hydrodynamics; the phase space of two-dimensional hydrodynamics is the set of all vorticity fields with a given set of values of these $Q_{k}$.

The information in these moments can be packaged into the vorticity distribution function $\rho(\lambda)$,

$$
\int_{-\infty}^{\infty} \rho(\lambda) \lambda^{k} d \lambda=\int \omega(x)^{k} d^{2} x
$$

Geometrically, $\rho(\lambda)$ is the perimeter of the contour curve of vorticity where $\omega(x)=$ $\lambda$. This can be written explicitly as

$$
\rho(\lambda)=\int \delta(\omega(x)-\lambda) d^{2} x
$$

The shape of these curves changes with time but not their perimeter.

In addition to these central functions, there may be additional conserved quantities such as momentum and angular momentum:

$$
P_{a}=\epsilon_{a b} \int x^{b} \omega(x) d^{2} x, \quad L=\frac{1}{2} \int x^{2} \omega(x) d^{2} x .
$$

But the presence of a boundary can break translational or rotation invariance, violating these conservation laws.

In spite of the presence of these infinite number of conservation laws, two dimensional hydrodynamics is far from being integrable: the phase space on which the $Q_{k}$ are constant is still infinite-dimensional. The crucial step in a statistical approach is the correct identification of entropy. This is ultimately defined by the canonical structure of the phase space: entropy of a macroscopic configuration is the log of the volume of the phase space corresponding to it. We would for example like to determine the volume of the phase space with a given set of values of moments $Q_{k}$. However, this is some infinite dimensional subspace of the space of all vorticity distributions. We need to find a way to approximate the phase space by a finite dimensional space: a 'regularization' or 'discretization' of the system. The entropy can then be determined within this discretized version of the theory and eventually a limit as the number of degrees of freedom goes to infinity can be taken.

We now describe an elegant discretization that preserves its symmetries (Lie algebra structure) and conservation laws. We will see that the problem of determining the entropy of a two dimensional flow can then be solved using ideas from Random matrix theory due to Wigner.

\section{Regularization}

It will be convenient to assume that the flow satisfies periodic boundary conditions in the plane. Our final result for entropy will be the same even if other boundary conditions are assumed, but the intermediate formulas seem simplest with periodic boundary conditions:

$$
\omega\left(x_{1}+L_{1}, x_{2}\right)=\omega\left(x_{1}, x_{2}\right), \quad \omega\left(x_{1}, x_{2}+L_{2}\right)=\omega\left(x_{1}, x_{2}\right) .
$$


We can then Fourier analyze vorticity:

$$
\begin{aligned}
& \omega_{m_{1} m_{2}}=\int_{0}^{L_{1}} \int_{0}^{L_{2}} \omega(x) e^{-2 \pi i \frac{m_{1} x_{1}}{L_{1}}} e^{-2 \pi i \frac{m_{2} x_{2}}{L_{2}}} d^{2} x, \\
& \omega(x)=\frac{1}{L_{1} L_{2}} \sum_{m_{1}, m_{2}=-\infty}^{\infty} \omega_{m_{1} m_{2}} e^{2 \pi i \frac{m_{1} x_{1}}{L_{1}}} e^{2 \pi i \frac{m_{2} x_{2}}{L_{2}}} .
\end{aligned}
$$

The hamiltonian and Poisson bracket are, in terms of these variables,

$$
H=\left(L_{1} L_{2}\right)^{2} \sum_{m \neq(0,0)} \frac{1}{m^{2}}\left|\omega_{m}\right|^{2}, \quad\left\{\omega_{m}, \omega_{n}\right\}=-\frac{2 \pi}{L_{1} L_{2}} \epsilon_{a b} m_{a} n_{b} \omega_{m+n} .
$$

The equations of motion become

$$
\frac{d \omega_{p}}{d t}=\sum_{m+n=p} \epsilon^{a b} m_{a} n_{b}\left[\frac{1}{m^{2}}-\frac{1}{n^{2}}\right] \omega_{m} \omega_{n}
$$

We are still dealing with a system with an infinite number of degrees of freedom. At first glance, ignoring all except the low momentum modes (i.e., keeping only $\left|m_{1}\right|,\left|m_{2}\right|<N$ for some $N$ ) looks like a reasonable 'regularization' of the problem: we would lose only information at very small length scales. However, such a naive truncation that simply ignores large $m_{1}, m_{2}$ modes would not be consistent: for example, the Poisson bracket of two low momentum modes could be a high momentum mode. We must modify the Poisson brackets so that the Poisson brackets of the modes we keep form a closed Lie algebra; as $N \rightarrow \infty$ this modification must dissappear and we must recover the original Lie algebra. Moreover the hamiltonian must be modified so that the equations don't mix high and low momentum modes.

A formalism along these lines is known in the literatured. The essential idea is to modify the coefficients in the Poisson brackets and hamiltonian so that they are periodic with period $N$; this way the algebra would be closed. There is a choice that satisfies the Jacobi identity, thus preserving the Lie algebra structure:

$$
\left\{\omega_{m}, \omega_{n}\right\}=\frac{1}{\theta} \sin \left[\theta\left(m_{1} n_{2}-m_{2} n_{1}\right)\right] \omega_{m+n \bmod N}, \quad \theta=\frac{2 \pi}{N} .
$$

We keep only a finite number of Fourier modes (we can assume that $N$ is odd $N=2 \nu+1)$,

$$
m_{1}, m_{2}, n_{1}, n_{2}=-\nu, \cdots 0,1, \cdots \nu .
$$

To be fair, the hamiltonian is truncated as well:

$$
H=\frac{1}{2} \sum_{\substack{m_{1}, m_{2}=-\nu \\ \lambda(m) \neq 0}}^{\nu} \frac{1}{\lambda(m)}\left|\omega_{m}\right|^{2} .
$$


It is natural to deform the eigenvalues of the Laplacian to $\lambda(m)=\left\{\frac{N}{2 \pi} \sin \left[\frac{2 \pi}{N} m_{1}\right]\right\}^{2}+$ $\left\{\frac{N}{2 \pi} \sin \left[\frac{2 \pi}{N} m_{2}\right]\right\}^{2}$. This would preserve the periodicity of the eigenvalue modulo $N$.

It is clear that as $N \rightarrow \infty, \theta \rightarrow 0$ and the structure constants of the Lie algebra tend to the original ones; so does the hamiltonian. The Lie algebra we obtain this way is in fact nothing but that of the Unitary group $U(N)$, the Lie algebra of hermitean matrices. Indeed the discretization procedure above has a natural interpretation in terms of non-commutative geometry; the Lie algebra is the algebra of derivations of the algebra of functions on a non-commutative torus. The constants $\lambda(m)$ in the hamiltonian above are the eigenvalues of the Laplace operator. However, we will not need this interpretation in what follows. Noncommutative geometry plays a deeper role in the three dimensional version of this theory, which one of us is still developing.

\section{Matrix Formulation}

It will be convenient to make a linear change of variables that will make the connection with hermitean matrices more explicit. Define $N \times N$ unitary matrices $U_{1}, U_{2}$ satisfying

$$
U_{1} U_{2}=e^{i \theta} U_{2} U_{1}
$$

Then, $U(m)=e^{-\frac{i}{2} m_{1} m_{2} \theta} U_{1}^{m_{1}} U_{2}^{m_{2}}$ satisfies $U^{\dagger}(m)=U(-m)$. To be specific we choose,

$$
U_{1}=\left(\begin{array}{cccc}
1 & 0 & \cdots & 0 \\
0 & e^{i \theta} & \cdots & 0 \\
\cdot & \cdot & \cdots & \cdot \\
\cdot & \cdot & \cdots & e^{i(N-1) \theta}
\end{array}\right), \quad U_{2}=\left(\begin{array}{cccccc}
0 & 0 & \cdots & 0 & 1 & \\
1 & 0 & \cdots & 0 & 0 & \\
\cdot & \cdot & \cdots & \cdot & \cdot & \\
0 & 0 & 0 & \cdots & 1 & 0
\end{array}\right) .
$$

(These matrices can be viewed as the coordinates on a non-commutative torus.) Now the Fourier coefficients of vorticity can be packaged in the hermitean matrix

$$
\hat{\omega}=\sum_{m} \omega_{m} U(m)
$$

(Recall that $\omega(x)$ being real implies that $\omega_{m}^{*}=\omega_{-m}$. This along with $U^{\dagger}(m)=$ $U(-m)$ implies that $\hat{\omega}^{\dagger}=\hat{\omega}$.) Now we can verify that

$$
\left\{\hat{\omega}_{a b}, \hat{\omega}_{c d}\right\}=i\left[\delta_{b c} \hat{\omega}_{a d}-\delta_{d a} \hat{\omega}_{c b}\right]
$$

These are the well-known commutation relations of the Lie algebra of $U(N)$ in the Weyl basis; this establishes the identification of our truncated Lie algebra. Moreover, we can see that the quantities

$$
\hat{Q}_{k}=\operatorname{tr} \hat{\omega}^{k}, \quad k=0,1, \cdots N-1
$$

are central in this algebra: they commute with any function of $\hat{\omega}$. In the limit as $N \rightarrow \infty, \theta \rightarrow 0$, these tend to the central functions $Q_{k}$ we started with. 
The hamiltonian becomes,

$$
H=\frac{1}{2} \hat{\omega}_{a b} \hat{\omega}_{c d} G_{a b c d}
$$

for some tensor $G_{a b c d}$ whose explicit form we do not need. The equation of motion are

$$
\frac{d \hat{\omega}}{d t}=i[\Omega, \hat{\omega}]
$$

where we have $\Omega_{a b}=G_{a b c d} \hat{\omega}_{c d}$. Thus time evolution is a sequence of unitary transformation so that the traces of powers of $\hat{\omega}$ are unchanged.

\section{Formula for Entropy}

Now it is clear that the information contained in the moments $\hat{Q}_{k}=\operatorname{tr} \hat{\omega}^{k}=$ $\sum_{j=1}^{N} \lambda_{j}^{k}$ is simply the spectrum $\left\{\lambda_{1}, \cdots, \lambda_{N}\right\}$ of the hermitean matrix $\hat{\omega}$. The phase space of the system is a vector space of finite dimension $N^{2}$. The submanifold of matrices with a fixed spectrum $\left\{\lambda_{1}, \cdots \lambda_{N}\right\}$ is compact and has dimension $N(N-1)$ : it is called the 'flag manifold' in algebraic geometry. There is a unique (up to a multiplicative constant) volume form on this submanifold invariant under the action of the unitary group. (This is the volume form induced by the symplectic structure associated to the Poisson brackets above.) The volume of microstates with a fixed value of moments $\hat{Q}_{k}$ are given by integrating this volume form.

The volume of this submanifold is well known

$$
V_{N}=C_{N} \prod_{1 \leq k<l \leq N}\left[\lambda_{k}-\lambda_{l}\right]^{2} .
$$

Here $C_{N}$ is a constant whose value we do not need. Thus the log of the volume of this manifold becomes

$$
\log V_{N}=2 \sum_{1 \leq k<l \leq N} \log \left|\lambda_{k}-\lambda_{l}\right|+\log C_{N}
$$

In terms of the density distribution of eigenvalues

$$
\rho_{N}(\lambda)=\frac{1}{N} \sum_{k=1}^{N} \delta\left(\lambda-\lambda_{k}\right),
$$

this becomes

$$
\log V_{N}=N^{2} \mathcal{P} \int \rho_{N}(\lambda) \rho_{N}\left(\lambda^{\prime}\right) \log \left|\lambda-\lambda^{\prime}\right| d \lambda d \lambda^{\prime}+\log C_{N}
$$

Here $\mathcal{P} \int$ denotes the principal value integral:

$$
\mathcal{P} \int f\left(\lambda, \lambda^{\prime}\right) d \lambda d \lambda^{\prime}=\lim _{\epsilon \rightarrow 0^{+}} \int_{\left|\lambda-\lambda^{\prime}\right|>\epsilon} f\left(\lambda, \lambda^{\prime}\right) d \lambda d \lambda^{\prime} .
$$


The advantage of this point of view is that it survives the limit $N \rightarrow \infty$, except for an undetermined additive constant.

Now, in the limit $N \rightarrow \infty$,

$$
\int \rho_{N}(\lambda) \lambda^{k} d \lambda \rightarrow \frac{1}{A} \int \rho(\lambda) \lambda^{k} d \lambda
$$

where $\rho(\lambda)=\int \delta(\omega(x)-\lambda) d^{2} x$ as we noted earlier. Also $A=\int d^{2} x$ is the total area of the region of the flow. Thus we see that $\lim _{N \rightarrow \infty} \rho_{N}(\lambda)=\bar{\rho}(\lambda)$, where $\bar{\rho}(\lambda)=\frac{1}{A} \rho(\lambda)$ is the normalized distribution of vorticity: $\int \bar{\rho}(\lambda) d \lambda=1$. We can now get the limiting form of the log of the volume of the submanifold with a given set of moments:

$$
\chi(\omega):=\lim _{N \rightarrow \infty} \frac{1}{N^{2}} \log V_{N}=\mathcal{P} \int \bar{\rho}(\lambda) \bar{\rho}\left(\lambda^{\prime}\right) \log \left|\lambda-\lambda^{\prime}\right| d \lambda d \lambda^{\prime} .
$$

(We drop the undetermined additive constant.) We can rewrite this directly in terms of vorticity:

$$
\chi(\omega)=\mathcal{P} \int \log |\omega(x)-\omega(y)| \frac{d^{2} x d^{2} y}{A^{2}} .
$$

This quantity should be viewed as the entropy of a vorticity distribution.

This formula for entropy is one of our main results. Our careful derivation by truncating the Lie algebra gives an entirely different result from those postulated in earlier analyses㧮, namely,

$$
\int \omega(x) \log |\omega(x)| d^{2} x
$$

\section{Semi-circular distribution}

As an application of this new notion of entropy we describe here the vortex distribution of maximum entropy with given total vorticity $Q_{1}$ and enstrophy $Q_{2}$. We need to maximize

$$
\chi(\rho)=\mathcal{P} \int \bar{\rho}(\lambda) \bar{\rho}\left(\lambda^{\prime}\right) \log \left|\lambda-\lambda^{\prime}\right| d \lambda d \lambda^{\prime}
$$

keeping the quantities $\int \bar{\rho}(\lambda) \lambda^{k} d \lambda$ for $k=0,1,2$ fixed. Introducing Lagrange multipliers $c_{0}, c_{1}, c_{2}$ for the constrained variational problem, we get

$$
2 \mathcal{P} \int \bar{\rho}\left(\lambda^{\prime}\right) \log \left|\lambda-\lambda^{\prime}\right| d \lambda^{\prime}=c_{0}+c_{1} \lambda+c_{2} \lambda^{2} .
$$

Differentating this to eliminate $c_{0}$ gives the singular integral equation

$$
2 \mathcal{P} \int \frac{\bar{\rho}\left(\lambda^{\prime}\right)}{\lambda-\lambda^{\prime}} d \lambda^{\prime}=c_{1}+2 c_{2} \lambda
$$


The equation is well known in the theory of random matrices; the solution is the 'semi-circular' distribution of Wigner

$$
\bar{\rho}(\lambda)=\frac{1}{2 \pi \sigma^{2}} \theta\left(\left|\lambda-\bar{Q}_{1}\right| \leq 2 \sigma\right) \sqrt{ }\left[4 \sigma^{2}-\left(\lambda-\bar{Q}_{1}\right)^{2}\right] .
$$

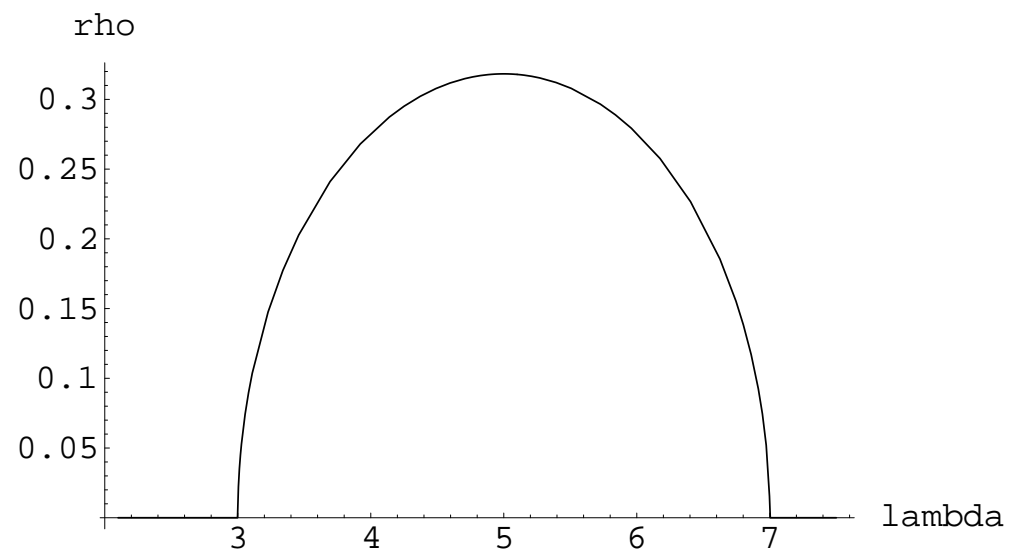

Fig. 1. Vorticity distribution function of maximum entropy with $\bar{Q}_{1}=5.0, \sigma=1.0$.

Here $\bar{Q}_{1}=\frac{1}{A} \int \omega(x) d^{2} x$ is the mean value of vorticity. The constant $\sigma$ is the standard deviation of the vorticity distribution and is determined by the enstrophy per unit area $\bar{Q}_{2}=\frac{1}{A} \int \omega(x)^{2} d^{2} x$ :

$$
\bar{Q}_{2}=\bar{Q}_{1}^{2}+\sigma^{2} .
$$

\section{Vorticity Distribution in a Cylindrically Symmetric Fluid}

As an applicaton of our ideas we determine the maximum entropy configuration of an axially symmetric fluid trapped between two concentric circles of radii $a_{1}<a_{2}$. The vorticity is a function only of the distance from the origin in the plane.

With axial symmetry, any function $\omega(r)$ of the radial distance alone will be a static solution of the Euler equations. We will seek the answer to the following question: among all cylindrically symmetric functions with given mean vorticity and enstropy, which one has the largest entropy? One should expect that a system subject to many perturbations will eventually settle down to this distribution.

Now, the vortex contours of an axisymmetric vorticity distribution are concentric circles. We should expect that the vorticity is monotonic with distance in the stable configuration. The vortex distribution is given by

$$
\rho(\lambda)=\int \delta(\rho(r)-\lambda) d^{2} x=2 \pi \int_{0}^{\infty} \delta(\omega(r)-\lambda) r d r .
$$


Let us introduce the variable $u=r^{2}$. Rather than think of the vorticity $\omega$ as a function of $r$ (or $u$ ), let us try to determine its inverse function; that is, $u$ as a function of $\omega$. Then we see that

$$
\rho(\lambda)=\pi\left|\frac{d u}{d \lambda}\right| .
$$

From the last section we know $\rho(\lambda)=A \bar{\rho}(\lambda)$ to be the semi-circular distribution. We can solve the above differential equation to determine the corresponding vorticity profile in parametric form:

$$
\omega=2 \sigma \sin \phi+\bar{Q}_{1}, \quad r^{2}=\frac{1}{2}\left[a_{1}^{2}+a_{2}^{2}\right] \pm\left[a_{2}^{2}-a_{1}^{2}\right] \frac{1}{\pi}\left[\phi+\frac{1}{2} \sin (2 \phi)\right],
$$

with the parameter $\phi$ taking the range of values

$$
-\frac{\pi}{2} \leq \phi \leq \frac{\pi}{2}
$$

There are two possible solutions with equal entropy: one with vorticity increasing with distance and the other with it decreasing. In most situations we would expect the vorticity to decrease with radial distance. We give a sample plot of this distribution below.

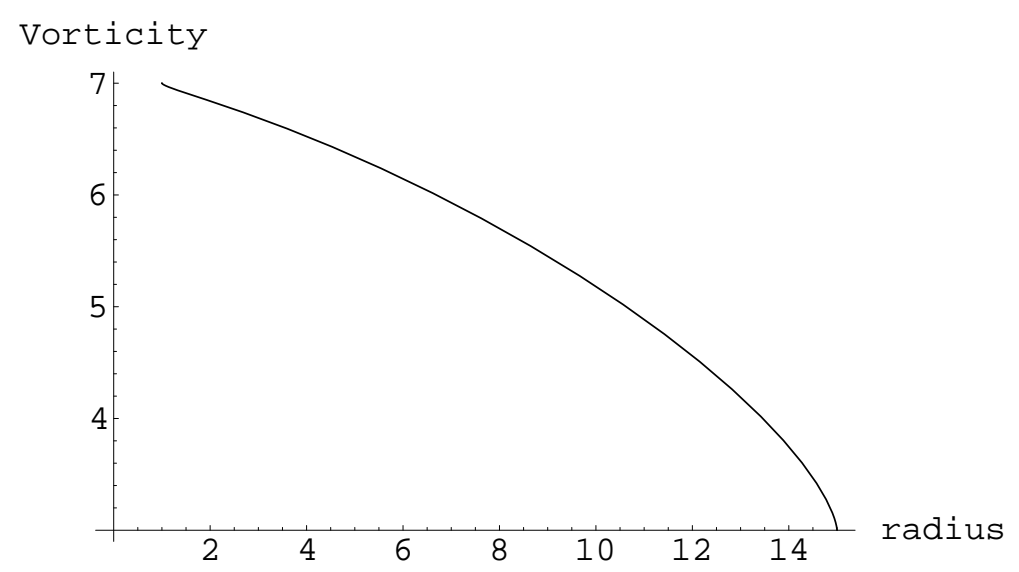

Fig. 2. Vorticity field of maximum entropy within radii $a_{1}=1.0, a_{2}=15$, mean vorticity $\bar{Q}_{1}=5.0$, and variance $\sigma^{2}=1.0$.

This could describe a vortex such as a hurricane, Jupiter's red spot or a tornado, to a reasonable approximation. A comparison with experimental measurements would be interesting.

\section{Acknowledgments}


The work of SVI was supported by the Dr. Nuala McGann Drescher Award funded by the State of New York/UUP Affirmative Action/Diversity Committee.

\section{References}

1. Dezhe Z. Jin and Daniel H.E. Dubin, Phys. Rev. Lett. 84, 1443 (2000); D.Z. Jin and D.H.E. Dubin, Phys. Rev. Lett. 80, 4434 (1998).

2. L. Onsager, "Statistical Hydrodynamics," Nuovo. Cim. Suppl. 6, 279 (1949).

3. R. Robert and J. Sommeria, "Statistical Equilibrium States for Two-dimensional Flows," J. Fluid Mech. 229, 291 (1991).

4. Jonathan Miller, "Statistical Mechanics of Euler Equations in Two Dimensions," Phys. Rev. Lett. 65, 2137 (1990); J. Miller, M.C. Cross and P.B. Weizmann, "Statistical Mechanics, Euler's Equation, and Jupiter's Red Spot," Phys. Rev. A 45, 2328 (1992).

5. M.L. Mehta, "Random Matrices," Academic Press (1991).

6. A. Polyakov, Nucl. Phys. B 396, 367 (1993); hep-th/9212145.

7. J. Hoppe, Int. J. Mod. Phys. A 4, 5235 (1989); D. B. Fairlie and C. K. Zachos, Phys. Lett. B 218, 203 (1989); D. B. Fairlie, P. Fletcher and C. K. Zachos, J. Math. Phys. 31, 1088 (1990); J.S. Dowker and A. Wolski, "Finite Model of Two-dimensional Ideal Hydrodynamics," Phys. Rev. A 46, 6417 (1992).

8. V.I. Arnol'd, "Sur La Geometrie differentielle des groupes de Lie de dimension infinie et ses applications a l'hydrodynamique des fluides parfaits," Ann. Inst. Fourier 16, 319 (1966). 